TRANSACTIONS OF THE

AMERICAN MATHEMATICAL SOCIETY

Volume 353, Number 5, Pages 1971-1984

S 0002-9947(01)02668-X

Article electronically published on January 4, 2001

\title{
THE LIMITS OF REFINABLE FUNCTIONS
}

\author{
GILBERT STRANG AND DING-XUAN ZHOU
}

\begin{abstract}
A function $\phi$ is refinable $(\phi \in S)$ if it is in the closed span of $\{\phi(2 x-k)\}$. This set $S$ is not closed in $L_{2}(\mathbb{R})$, and we characterize its closure. A necessary and sufficient condition for a function to be refinable is presented without any information on the refinement mask. The Fourier transform of every $f \in \bar{S} \backslash S$ vanishes on a set of positive measure. As an example, we show that all functions with Fourier transform supported in $\left[-\frac{4}{3} \pi, \frac{4}{3} \pi\right]$ are the limits of refinable functions. The relation between a refinable function and its mask is studied, and nonuniqueness is proved. For inhomogeneous refinement equations we determine when a solution is refinable. This result is used to investigate refinable components of multiple refinable functions. Finally, we investigate fully refinable functions for which all translates (by any real number) are refinable.
\end{abstract}

\section{Introduction and Main Results}

The central equation in wavelet analysis is the refinement equation for the scaling function $\phi$ :

$$
\phi(x)=\sum_{k=0}^{N} a(k) \phi(2 x-k) .
$$

In approximation theory, the sequence $\{a(k)\}$ is the mask. In signal processing these are the coefficients of a lowpass filter.

For a given mask $\{a(k)\}$, wavelet theory yields the properties of the family $\{\phi(x-k)\}$. We can determine whether these translates form a Riesz basis of a subspace in $L_{2}(\mathbb{R})$, whether this basis is orthogonal, and which polynomials $1, x, \cdots, x^{p-1}$ are linear combinations of the translates. This theory is summarized in [3] and [14]. What we do not know is how to choose the mask $\{a(k)\}$ so that $\phi(x)$ is close to a given function $f(x)$.

This "inverse problem" arises naturally in applications. We want to recognize objects whose shape is indicated by $f(x)$. We hope that a scaling function of similar shape will allow us to identify a good match. The thesis of Chapa 2 made a start on this problem using band-limited scaling functions. In that case the Fourier transform $\hat{\phi}(\xi)$ has compact support and the sequence $\{a(k)\}$ is infinite.

Received by the editors May 15, 1998 and, in revised form, November 3, 1999.

2000 Mathematics Subject Classification. Primary 42C40, 41A25; Secondary 65F15.

Key words and phrases. Refinable function, Fourier transform, band-limited function, refinement mask, inhomogeneous refinement equation, multiple refinable function, fully refinable function.

Research supported in part by Research Grants Council of Hong Kong. 
We want to start again, by answering this preliminary question: What is the closure of the set $S$ of all refinable functions in $L_{2}(\mathbb{R})$ ? A solution to (1.1) is a refinable function. More generally, we say that

$$
\phi \text { is refinable }(\phi \in S) \quad \text { if and only if } \quad \phi(x) \in \overline{\operatorname{span}}\{\phi(2 x-k): k \in \mathbb{Z}\} .
$$

Thus an infinite mask is allowed. The refinability is better understood in the frequency domain. To see this, we need the following characterization of closed shift-invariant subspaces in $L_{2}(\mathbb{R})$ given explicitly by de Boor, DeVore, and Ron [1], based on doubly-invariant spaces discussed in [7]. Each such subspace is associated with a function $\phi$ in $L_{2}(\mathbb{R})$. The subspace $S_{2}(\phi)=\overline{\operatorname{span}}\{\phi(x-k): k \in \mathbb{Z}\}$ is

$$
S_{2}(\phi)=\left\{f \in L_{2}(\mathbb{R}): \hat{f}(\xi)=\tau(\xi) \hat{\phi}(\xi) \text { for a } 2 \pi \text {-periodic function } \tau(\xi)\right\} \text {. }
$$

It follows that $\phi \in S$ if and only if, for some $2 \pi$-periodic function $\tilde{a}$,

$$
\hat{\phi}(2 \xi)=\tilde{a}(\xi) \hat{\phi}(\xi), \quad \text { for almost every } \xi .
$$

Let us turn to the set of all refinable functions. We wondered at first whether this set $S$ is closed. We will show that it is not closed, and Theorem 1 will describe its closure $\bar{S}$. The crucial questions involve the zeros of the Fourier transform. Recall from (1.4) that $\phi \in S$ satisfies $\hat{\phi}(2 \xi)=\tilde{a}(\xi) \hat{\phi}(\xi)$ for some $2 \pi$-periodic function. In the inverse direction, $f$ will be refinable if $\hat{f}(2 \xi) / \hat{f}(\xi)$ happens to be $2 \pi$-periodic, and $\hat{f}(\xi)$ is never zero. Then $f$ will solve equation (1.1) with symbol of the mask given by

$$
\tilde{a}(\xi)=\frac{\hat{f}(2 \xi)}{\hat{f}(\xi)} .
$$

But if $\hat{f}(\xi)$ has zeros (which is typical!), we have to consider their relation to the zeros of $\hat{f}(2 \xi)$. This eventually leads to our characterization of the closure of $S$ :

Theorem 1. A function $f$ lies in $\bar{S}$, the closure of $S$ in $L_{2}(\mathbb{R})$, if and only if, for any positive integers $j$ and $k$,

$$
\hat{f}\left(2^{j}(\xi+2 k \pi)\right) \hat{f}(\xi)=\hat{f}\left(2^{j} \xi\right) \hat{f}(\xi+2 k \pi) \quad \text { for almost every } \xi
$$

As an example, the function $f \in L_{2}(\mathbb{R})$ given by

$$
\hat{f}(\xi)= \begin{cases}1, & \text { if } \xi \in\left(-\frac{4}{3} \pi,-\frac{2}{3} \pi\right) \cup\left(\frac{2}{3} \pi, \frac{4}{3} \pi\right), \\ 0, & \text { otherwise, }\end{cases}
$$

is in $\bar{S}$, but is not refinable. The condition (1.5) certainly holds in the band-limited case when $\hat{f}(\xi)$ is supported in $\left[-\frac{4}{3} \pi, \frac{4}{3} \pi\right]$, because for $\xi$ in this interval we have

$$
\left|2^{j}(\xi+2 k \pi)\right| \geq 2(2 \pi-|\xi|) \geq \frac{4}{3} \pi
$$

Then both sides of (1.5) are identically zero and $f \in \bar{S}$. Section 3 will show that if $b>\frac{4}{3} \pi$, there are functions with $\hat{f}(\xi)$ supported on $[-b, b]$ for which $(1.5)$ does not hold.

Our second main result is a lower bound on the distance $d(f, S)$ from $f$ to $S$ :

$$
d(f, S)=\inf \left\{\|f-\phi\|_{2}: \phi \in S\right\}
$$


From the characterization of Theorem 1, it is natural to measure this distance in terms of the family of functions

$$
D_{j, k}(f)(\xi):=\hat{f}\left(2^{j}(\xi+2 k \pi)\right) \hat{f}(\xi)-\hat{f}\left(2^{j} \xi\right) \hat{f}(\xi+2 k \pi) .
$$

Theorem 2. Let $f$ be a nonzero function in $L_{2}(\mathbb{R})$. Then

$$
d(f, S) \geq \frac{\sqrt{2}-1}{12 \pi\|f\|_{2}} \sup _{k \in \mathbb{N}}\left\{\sum_{j=1}^{\infty}\left\|D_{j, k}(f)\right\|_{1}\right\} .
$$

In the proof of Theorem 1 , the family of sets $\left\{K_{j}(f)\right\}$ defined for functions as

$$
K_{j}(f):=\left\{\xi \in[-\pi, \pi): \hat{f}\left(2^{j}(\xi+2 l \pi)\right) \neq 0 \text { for some } l \in \mathbb{Z}\right\}
$$

plays an essential role. In terms of these sets, the proof of Theorem 1 also provides a characterization of refinable functions.

Theorem 3. Let $f \in \bar{S}$. Then $f$ lies in $S$ if and only if the set $\bigcup_{j=1}^{\infty} K_{j}(f) \backslash K_{0}(f)$ has measure zero.

Corollary 1. If $f \in \bar{S} \backslash S$, then $\hat{f}(\xi)$ vanishes on a set of positive measure.

The final sections of the paper deal with smaller points in the theory of refinable functions:

Section 4: Nonuniqueness of the mask.

Section 5. Refinable solutions to inhomogeneous refinement equations.

Section 6. Multiple refinable functions (leading to multiwavelets).

Section 7. Fully refinable functions (all translates $\phi(x-t)$ are refinable).

\section{Proof of the Main Results}

In this section we prove the main results. Recall the characterization (1.3).

Proof of Theorem 1. Necessity of (1.5). Suppose that there is a sequence $\left\{\phi_{n}\right\} \subset S$ such that $\left\|\phi_{n}-f\right\|_{2} \rightarrow 0$ as $n \rightarrow \infty$. Then $\left\|\hat{\phi}_{n}-\hat{f}\right\|_{2} \rightarrow 0$. Hence there is a subsequence $\left\{\hat{\phi}_{n_{k}}(\xi)\right\}$ such that

$$
\lim _{k \rightarrow \infty} \hat{\phi}_{n_{k}}(\xi)=\hat{f}(\xi)
$$

almost everywhere. By replacing $\left\{\phi_{n}\right\}$ with this subsequence, we may assume that

$$
\lim _{n \rightarrow \infty} \hat{\phi}_{n}(\xi)=\hat{f}(\xi), \quad \forall \xi \in \mathbb{R} \backslash T,
$$

where $T$ is a set of measure zero (null set).

Since $\phi_{n}$ is refinable, by (1.3) there is a $2 \pi$-periodic function $\tilde{a}_{n}(\xi)$ such that

$$
\hat{\phi}_{n}(2 \xi)=\tilde{a}_{n}(\xi) \hat{\phi}_{n}(\xi)
$$

almost everywhere. By recursion this implies for all $n$ and all $j=1,2, \cdots$ that

$$
\hat{\phi}_{n}\left(2^{j} \xi\right)=\tilde{a}_{n}\left(2^{j-1} \xi\right) \cdots \tilde{a}_{n}(\xi) \hat{\phi}_{n}(\xi), \quad \forall \xi \in \mathbb{R} \backslash T^{\prime},
$$

where $T^{\prime}$ is another null set. Then $T_{j}=\left(T^{\prime}+2 \pi \mathbb{Z}\right) \cup\left(2^{-j} T+2 \pi \mathbb{Z}\right) \cup(T+2 \pi \mathbb{Z})$ is also a null set. Suppose $\xi \in \mathbb{R} \backslash T_{j}$.

Let $k \in \mathbb{N}$. If $\hat{f}(\xi+2 k \pi)=\hat{f}(\xi)=0$, then (1.5) holds trivially.

If $\hat{f}(\xi+2 l \pi) \neq 0$ for $l=0$ or $l=k$, then (2.1) and (2.3) imply that

$$
\lim _{n \rightarrow \infty} \tilde{a}_{n}\left(2^{j-1} \xi\right) \cdots \tilde{a}_{n}(\xi)=\hat{f}\left(2^{j}(\xi+2 l \pi)\right) / \hat{f}(\xi+2 l \pi) .
$$


By the $2 \pi$-periodicity, taking the limits in (2.1) and (2.3) again, we have

$$
\hat{f}\left(2^{j}(\xi+2 p \pi)\right)=\frac{\hat{f}\left(2^{j}(\xi+2 l \pi)\right)}{\hat{f}(\xi+2 l \pi)} \hat{f}(\xi+2 p \pi), \quad \forall p \in \mathbb{Z} .
$$

In particular, the choice $p \in\{0, k\} \backslash\{l\}$ implies (1.5).

Thus (1.5) is true for every $k \in \mathbb{N}$ and this $\xi \in \mathbb{R} \backslash T_{j}$.

Since the set $T_{j}$ has measure zero, (1.5) holds almost everywhere. This proves the necessity of (1.5).

Sufficiency. Suppose that (1.5) is true. It is still true if we replace $\xi$ by $2^{m}(\xi+2 l \pi)$ and $k$ by $2^{m} k$, for $m \in \mathbb{N}$ and $l \in \mathbb{Z}$. Now change $j+m$ back to $j$, and $k+l$ back to $k$. The result is

$$
\hat{f}\left(2^{j}(\xi+2 k \pi)\right) \hat{f}\left(2^{m}(\xi+2 l \pi)\right)=\hat{f}\left(2^{j}(\xi+2 l \pi)\right) \hat{f}\left(2^{m}(\xi+2 k \pi)\right)
$$

for any $j, m \in \mathbb{N}, k, l \in \mathbb{Z}$ and every $\xi \in \mathbb{R} \backslash T$, where $T$ is a null set.

Let us define a sequence $\left\{\phi_{n}\right\}$ of refinable functions tending to $f$.

Let $M_{j}(f)$ be the union of the sets $K_{0}(f), \cdots, K_{j-1}(f)$ defined in (1.7):

$$
\begin{aligned}
M_{j}(f): & =\bigcup_{i=0}^{j-1} K_{i}(f) \\
& =\left\{\xi \in[-\pi, \pi): \hat{f}\left(2^{i}(\xi+2 l \pi)\right) \neq 0 \text { for some } 0 \leq i<j \text { and } l \in \mathbb{Z}\right\} .
\end{aligned}
$$

Naturally, we set $M_{\infty}(f)$ as

$$
M_{\infty}(f)=\bigcup_{i=0}^{\infty} K_{i}(f)=\bigcup_{j=1}^{\infty} M_{j}(f) .
$$

For $\xi \in K_{0}(f)$, define

$$
\hat{\phi}_{n}(\xi+2 k \pi)=\hat{f}(\xi+2 k \pi), \quad \forall k \in \mathbb{Z} .
$$

For $j \in \mathbb{N}$ and $\xi \in K_{j}(f) \backslash M_{j}(f)$, define

$$
\hat{\phi}_{n}(\xi+2 k \pi)=\hat{f}\left(2^{j}(\xi+2 k \pi)\right) / n, \quad \forall k \in \mathbb{Z} .
$$

For $\xi \in[-\pi, \pi) \backslash M_{\infty}(f)$, define

$$
\hat{\phi}_{n}(\xi+2 k \pi)=0, \quad \forall k \in \mathbb{Z} .
$$

Thus, $\hat{\phi}_{n}(\xi)$ has been defined for all $\xi$.

We first show that $\hat{\phi}_{n}(\xi) \rightarrow \hat{f}(\xi)$ in $L_{2}(\mathbb{R})$. By equations (2.5) and (2.7),

$$
\hat{\phi}_{n}(\xi+2 k \pi)-\hat{f}(\xi+2 k \pi)=0, \quad \forall \xi \in K_{0}(f) \cup\left([-\pi, \pi) \backslash M_{\infty}(f)\right), \quad k \in \mathbb{Z} .
$$

Hence

$$
\begin{aligned}
\left\|\hat{\phi}_{n}-\hat{f}\right\|_{2}^{2} & =\sum_{j=1}^{\infty} \int_{K_{j}(f) \backslash M_{j}(f)} \sum_{k \in \mathbb{Z}}\left|\hat{\phi}_{n}(\xi+2 k \pi)-\hat{f}(\xi+2 k \pi)\right|^{2} d \xi \\
& =\sum_{j=1}^{\infty} \int_{K_{j}(f) \backslash M_{j}(f)} \sum_{k \in \mathbb{Z}}\left|\hat{f}\left(2^{j}(\xi+2 k \pi)\right) / n\right|^{2} d \xi \\
& \leq \sum_{j=1}^{\infty} \int_{\mathbb{R}}\left|\hat{f}\left(2^{j} \xi\right)\right|^{2} d \xi / n^{2} \\
& =\|\hat{f}\|_{2}^{2} / n^{2} \rightarrow 0 .
\end{aligned}
$$


Therefore, $\phi_{n} \in L_{2}(\mathbb{R})$ and $\lim _{n \rightarrow \infty}\left\|\phi_{n}-f\right\|_{2}=0$.

Next we show that $\phi_{n}$ is refinable, by constructing $\tilde{a}_{n}(\xi)$ on $[-\pi, \pi)$ such that

$$
\hat{\phi}_{n}(2(\xi+2 k \pi))=\tilde{a}_{n}(\xi) \hat{\phi}_{n}(\xi+2 k \pi), \quad \forall k \in \mathbb{Z}, \quad \xi \in[-\pi, \pi) \backslash T .
$$

Let $j \in \mathbb{N} \cup\{0\}$ and $\xi \in K_{j}(f) \backslash M_{j}(f)$, and furthermore choose $k_{\xi} \in \mathbb{Z}$ such that $\hat{f}\left(2^{j}\left(\xi+2 k_{\xi} \pi\right)\right) \neq 0$. Define

$$
\tilde{a}_{n}(\xi)= \begin{cases}\hat{\phi}_{n}\left(2\left(\xi+2 k_{\xi} \pi\right)\right) / \hat{f}\left(\xi+2 k_{\xi} \pi\right), & \text { if } j=0, \\ n \hat{\phi}_{n}\left(2\left(\xi+2 k_{\xi} \pi\right)\right) / \hat{f}\left(2^{j}\left(\xi+2 k_{\xi} \pi\right)\right), & \text { if } j \in \mathbb{N} .\end{cases}
$$

For $\xi \in[-\pi, \pi) \backslash M_{\infty}(f)$, we can define $\tilde{a}_{n}(\xi)$ arbitrarily.

Let us now verify the refinement relation (2.8), first for $\xi \in M_{\infty}(f) \backslash T$.

Let $j \in \mathbb{N} \cup\{0\}$ and $\xi \in K_{j}(f) \backslash M_{j}(f) \backslash T$. For every $k \in \mathbb{Z}$,

$$
\tilde{a}_{n}(\xi) \hat{\phi}_{n}(\xi+2 k \pi)=\frac{\hat{\phi}_{n}\left(2\left(\xi+2 k_{\xi} \pi\right)\right)}{\hat{f}\left(2^{j}\left(\xi+2 k_{\xi} \pi\right)\right)} \hat{f}\left(2^{j}(\xi+2 k \pi)\right) .
$$

To see that this equals $\hat{\phi}_{n}(2(\xi+2 k \pi))$, write $2 \xi$ as $\eta+2 s \pi$ with $\eta \in[-\pi, \pi)$ and $s \in \mathbb{Z}$. Then, if $\eta \notin M_{\infty}(f)$,

$$
\hat{\phi}_{n}(2(\xi+2 l \pi))=\hat{\phi}_{n}(\eta+2 s \pi+4 l \pi)=0, \quad \forall l \in \mathbb{Z}
$$

Hence the right-hand side of $(2.9)$ equals $\hat{\phi}_{n}(2(\xi+2 k \pi))$ in this case.

If $\eta \in K_{0}(f)$, then

$$
\hat{\phi}_{n}(2(\xi+2 l \pi))=\hat{\phi}_{n}(\eta+2 s \pi+4 l \pi)=\hat{f}(\eta+2 s \pi+4 l \pi)=\hat{f}(2(\xi+2 l \pi)) .
$$

Hence the right-hand side of $(2.9)$ equals $\hat{\phi}_{n}(2(\xi+2 k \pi))$ again by the condition (2.4).

If $\eta \in K_{m}(f) \backslash M_{m}(f)$ for some $m \in \mathbb{N}$, then

$$
\begin{aligned}
\hat{\phi}_{n}(2(\xi+2 l \pi)) & =\hat{\phi}_{n}(\eta+2 s \pi+4 l \pi)=\hat{f}\left(2^{m}(\eta+2 s \pi+4 l \pi)\right) / n \\
& =\hat{f}\left(2^{m+1}(\xi+2 l \pi)\right) / n .
\end{aligned}
$$

Hence the right-hand side of $(2.9)$ equals $\hat{\phi}_{n}(2(\xi+2 k \pi))$ in this final case by the condition (2.4).

Thus, the refinement relation (2.8) has been proved for $\xi \in M_{\infty}(f) \backslash T$.

Next we consider $\xi \in[-\pi, \pi) \backslash M_{\infty}(f) \backslash T$. Here we have

$$
\hat{\phi}_{n}(\xi+2 l \pi)=0, \quad \forall l \in \mathbb{Z} .
$$

Let us show that $\hat{\phi}_{n}(2(\xi+2 l \pi))=0$ for every $l \in \mathbb{Z}$. Write $2 \xi$ as $\eta+2 s \pi$ again with $\eta \in[-\pi, \pi)$ and $s \in \mathbb{Z}$.

If $\eta \notin M_{\infty}(f)$, then

$$
\hat{\phi}_{n}(2(\xi+2 l \pi))=\hat{\phi}_{n}(\eta+2 s \pi+4 l \pi)=0, \quad \forall l \in \mathbb{Z} .
$$

If $\eta \in K_{0}(f)$, then $\xi \notin K_{1}(f)$ implies that for every $l \in \mathbb{Z}$,

$$
\hat{\phi}_{n}(2(\xi+2 l \pi))=\hat{f}(\eta+2 s \pi+4 l \pi)=\hat{f}(2(\xi+2 l \pi))=0 .
$$

If $\eta \in K_{j}(f) \backslash M_{j}(f)$ for some $j \in \mathbb{N}$, then

$$
\begin{aligned}
\hat{\phi}_{n}(2(\xi+2 l \pi)) & =\hat{\phi}_{n}(\eta+2 s \pi+4 l \pi)=\hat{f}\left(2^{j}(\eta+2 s \pi+4 l \pi)\right) / n \\
& =\hat{f}\left(2^{j+1}(\xi+2 l \pi)\right) / n=0, \quad \forall l \in \mathbb{Z},
\end{aligned}
$$

since $\xi \notin K_{j+1}(f)$. 
Thus, in all three cases,

$$
\hat{\phi}_{n}(2(\xi+2 l \pi))=0, \quad \forall l \in \mathbb{Z} .
$$

Therefore, the refinement relation (2.8) holds true on $[\pi, \pi) \backslash T$. Hence $\phi_{n}$ is refinable; it lies in $S$. Then $\lim \left\|\phi_{n}-f\right\|_{2}=0$ tells us that $f$ lies in the closure of $S$.

Now that we have proved Theorem 1, the proof of Theorem 2 follows quickly.

Proof of Theorem 2. For $\phi \in S$, Theorem 1 gives $D_{j, k}(\phi)(\xi)=0$ almost everywhere. Then

$$
\begin{aligned}
\left\|D_{j, k}(f)\right\|_{1}=\left\|D_{j, k}(f)-D_{j, k}(\phi)\right\|_{1} \\
=\int_{\mathbb{R}} \mid\left[\hat{f}\left(2^{j}(\xi+2 k \pi)\right)-\hat{\phi}\left(2^{j}(\xi+2 k \pi)\right)\right] \hat{f}(\xi) \\
\quad+\hat{\phi}\left(2^{j}(\xi+2 k \pi)\right)[\hat{f}(\xi)-\hat{\phi}(\xi)] \\
\quad+\hat{\phi}\left(2^{j} \xi\right)[\hat{\phi}(\xi+2 k \pi)-\hat{f}(\xi+2 k \pi)] \\
\quad+\hat{f}(\xi+2 k \pi)\left[\hat{\phi}\left(2^{j} \xi\right)-\hat{f}\left(2^{j} \xi\right)\right] \mid d \xi .
\end{aligned}
$$

Applying the Schwarz inequality, we get

$$
\begin{aligned}
\left\|D_{j, k}(f)\right\|_{1} & \leq 2\left(\int\left|\hat{f}\left(2^{j} \xi\right)-\hat{\phi}\left(2^{j} \xi\right)\right|^{2} d \xi\right)^{1 / 2}\|\hat{f}\|_{2}+2\left(\int\left|\hat{\phi}\left(2^{j} \xi\right)\right|^{2} d \xi\right)^{1 / 2}\|\hat{f}-\hat{\phi}\|_{2} \\
& =2^{1-j / 2}\|\hat{f}-\hat{\phi}\|_{2}\left(\|\hat{f}\|_{2}+\|\hat{\phi}\|_{2}\right) .
\end{aligned}
$$

For each $k$ we sum over $j \in \mathbb{N}$ :

$$
\|f-\phi\|_{2}\left(\|f\|_{2}+\|\phi\|_{2}\right) \geq \frac{\sqrt{2}-1}{4 \pi} \sum_{j=1}^{\infty}\left\|D_{j, k}(f)\right\|_{1} .
$$

In computing the distance $d(f, S)$ we may restrict to $\phi \in S$ with $\|\phi\|_{2} \leq 2\|f\|_{2}$, since otherwise $\|\phi-f\| \geq\|0-f\|$. Then (2.10), for each $k$, yields the lower bound on $d(f, S)$ in Theorem 2 :

$$
d(f, S) 3\|f\|_{2} \geq \frac{\sqrt{2}-1}{4 \pi} \sum_{j=1}^{\infty}\left\|D_{j, k}(f)\right\|_{1} .
$$

The proof of Theorem 2 is complete.

Remark on condition (1.5). If the Fourier transform of a refinable function were never zero, division would be allowed and everything would become easy:

$$
\begin{gathered}
\frac{\hat{f}(2 \xi)}{\hat{f}(\xi)} \quad \text { is periodic by the refinement equation, and } \\
\frac{\hat{f}\left(2^{j} \xi\right)}{\hat{f}(\xi)}=\frac{\hat{f}\left(2^{j} \xi\right)}{\hat{f}\left(2^{j-1} \xi\right)} \cdots \frac{\hat{f}(2 \xi)}{\hat{f}(\xi)} \quad \text { is periodic by induction. }
\end{gathered}
$$

Condition (1.5) is simply the periodicity of $\hat{f}\left(2^{j} \xi\right) / \hat{f}(\xi)$ after multiplication to clear out the (possibly zero!) denominators. 
Since the periodicity (2.13) for all $j$ follows from $(2.12)$ for $j=1$, it is natural to ask whether this is also true in condition (1.5). Must we impose this condition for all $j \in \mathbb{N}$ ?

The following example shows that we must.

Example 1. Let $\hat{f}(\xi)=1$ for

$$
\xi \in(-\pi,-\pi / 2) \cup\left(-\pi / 2^{m+1},-\pi / 2^{m+2}\right) \cup\left(2 \pi-\pi / 2^{m+1}, 2 \pi-\pi / 2^{m+2}\right)
$$

and zero elsewhere. Then (1.5) holds for $j=1, \cdots, m$ and all $k$, but not for $j=m+1$ and $k=1$.

Proof. If $\xi>0$, then

$$
\hat{f}(\xi+2 k \pi)=\hat{f}\left(2^{j}(\xi+2 k \pi)\right)=0 \quad \text { for all } j, k \in \mathbb{N} .
$$

If $\xi<-\pi$, then

$$
\hat{f}(\xi)=\hat{f}\left(2^{j} \xi\right)=0 \quad \text { for all } j \in \mathbb{N} \text {. }
$$

If $-\pi<\xi<0$, then

$$
\hat{f}\left(2^{j}(\xi+2 k \pi)\right)=0 \quad \text { for all } j, k \in \mathbb{N} .
$$

If $-\pi<\xi<-\pi / 2^{m+1}$ or $-\pi / 2^{m+2}<\xi<0$, then

$$
\hat{f}(\xi+2 k \pi)=0 \quad \text { for all } k \in \mathbb{N} .
$$

If $-\pi<\xi<0$, then

$$
\hat{f}(\xi+2 k \pi)=0 \quad \text { for all } k \geq 2 .
$$

Thus we only need to check condition (1.5) for $-\pi / 2^{m+1}<\xi<-\pi / 2^{m+2}$ and $k=1$. In this case, $\hat{f}(\xi+2 \pi)=1$. For $j=1, \cdots, m$ we have $\hat{f}\left(2^{j} \xi\right)=0$, which implies (1.5). However, $\hat{f}\left(2^{m+1} \xi\right)=1$, which contradicts (1.5) for $k=1$.

\section{BAnd-Limited Functions}

Let $X_{b}$ be the set of band-limited functions with frequencies $\xi$ restricted to the band $[-b, b]$ :

$$
X_{b}:=\left\{f \in L_{2}(\mathbb{R}): \operatorname{supp} \hat{f} \subset[-b, b]\right\} .
$$

We observed in the introduction that $X_{b} \subset \bar{S}$ for $b \leq \frac{4}{3} \pi$. The converse is also true.

Theorem 4. $X_{b} \subset \bar{S}$ if and only if $b \leq \frac{4}{3} \pi$.

Proof. If $b>\frac{4}{3} \pi$, let $f \in L_{2}(\mathbb{R})$ be given by its Fourier transform as

$$
\hat{f}(\xi)= \begin{cases}1, & \text { if }|\xi|<B:=\min \{b, 2 \pi\} \\ 0, & \text { otherwise. }\end{cases}
$$

Then for $j=1, k=1,-B<\xi<-\frac{4}{3} \pi$, we have

$$
\hat{f}(2(\xi+2 \pi))=\hat{f}(\xi)=1 \text { but } \hat{f}(2 \xi)=0 .
$$

Hence (1.5) does not hold on the interval $\left(-B,-\frac{4}{3} \pi\right)$ for $j=1, k=1$. Thus, $f \in X_{b} \backslash \bar{S}$. 
Now we show that some functions are not refinable but are the limits of refinable functions. The example in the introduction was

$$
\hat{f}(\xi)= \begin{cases}1, & \text { if } \xi \in\left(-\frac{4}{3} \pi,-\frac{2}{3} \pi\right) \cup\left(\frac{2}{3} \pi, \frac{4}{3} \pi\right), \\ 0, & \text { otherwise. }\end{cases}
$$

Corollary 2. Let $f \in X_{\frac{4}{3} \pi}$, and let $K(f):=\{\xi \in \mathbb{R}: \hat{f}(\xi) \neq 0\}$ be the support of $\hat{f}$. Then $f$ is refinable if and only if $\frac{1}{2} K(f) \subset K(f)$ up to a null set.

Proof. By Theorem 4, $f \in \bar{S}$.

Observe that for $f \in X_{\frac{4}{3} \pi}$ and $j \in \mathbb{N}$,

$$
K_{j}(f)=\left\{\xi \in[-\pi, \pi): \hat{f}\left(2^{j} \xi\right) \neq 0\right\}=2^{-j} K(f) \subset\left[-\frac{2}{3} \pi, \frac{2}{3} \pi\right] .
$$

Also,

$$
K_{0}(f) \cap\left(-\frac{2}{3} \pi, \frac{2}{3} \pi\right)=K(f) \cap\left(-\frac{2}{3} \pi, \frac{2}{3} \pi\right) .
$$

Then our conclusion follows from Theorem 3 .

Combining Theorem 4 and Corollary 2, we know that every nonzero function in $X_{\frac{4}{3} \pi}$ whose Fourier transform vanishes on $\left[-\frac{2}{3} \pi, \frac{2}{3} \pi\right]$ lies in $\bar{S} \backslash S$.

\section{Refinable Functions and Masks: Nonuniqueness}

We apply the characterization of refinable functions in Theorem 3 to show that the function may not determine the mask (and vice versa).

First, we show that the $2 \pi$-periodic symbol of the mask $\tilde{a}(\xi)$ is sometimes not unique.

Theorem 5. Let $\phi$ be a nonzero refinable function in $L_{2}(\mathbb{R})$, and let $K_{0}(\phi)$ be defined by (1.7). Then the refinement mask $\tilde{a}(\xi)$ satisfying (1.4) is unique (up to a null set) if and only if meas $\left(K_{0}(\phi)\right)=2 \pi$, i.e., for almost every $\xi \in[-\pi, \pi)$ there is some $k_{\xi} \in \mathbb{Z}$ such that $\hat{\phi}\left(\xi+2 k_{\xi} \pi\right) \neq 0$.

Proof. The sufficiency is clear, since $\tilde{a}(\xi)$ is determined for $\xi \in K_{0}(\phi)$ by

$$
\tilde{a}(\xi)=\hat{\phi}\left(2\left(\xi+2 k_{\xi} \pi\right)\right) / \hat{\phi}\left(\xi+2 k_{\xi} \pi\right),
$$

which defines $\tilde{a}(\xi)$ uniquely up to a null set.

For the necessity, suppose to the contrary that meas $\left(K_{0}(\phi)\right)<2 \pi$. Then the measure of the set $\left([-\pi, \pi) \backslash M_{\infty}(\phi)\right)$ is positive by Theorem 3 .

Let $\tilde{a}(\xi)$ be the symbol of a refinement mask satisfying (1.4). Choose $\tilde{b}(\xi)$ to be a $2 \pi$-periodic function satisfying

$$
\tilde{b}(\xi)=\tilde{a}(\xi), \quad \forall \xi \in K_{0}(\phi) .
$$

Then we can see that for almost every $\xi \in[-\pi, \pi)$,

$$
\hat{\phi}(2(\xi+2 k \pi))=\tilde{b}(\xi) \hat{\phi}(\xi+2 k \pi), \quad \forall k \in \mathbb{Z} .
$$

In fact, for $\xi \in[-\pi, \pi) \backslash M_{\infty}(\phi)$,

$$
\hat{\phi}(2(\xi+2 k \pi))=\hat{\phi}(\xi+2 k \pi)=0, \quad \forall k \in \mathbb{Z},
$$

while $\tilde{b}(\xi)=\tilde{a}(\xi)$ for $\xi \in K_{0}(\phi)$. Hence the refinement relation is reduced to (1.4). 
Note that meas $\left(\left([-\pi, \pi) \backslash M_{\infty}(\phi)\right) \cup K_{0}(\phi)\right)=2 \pi$ by Theorem 3 . The function $\tilde{b}(\xi)$ is also the symbol of a refinement mask for $\phi$. Thus the mask is not unique.

Second, we show that the refinable function is never unique, given a refinement mask.

The classical approach begins with a sequence $\{a(k)\}$ satisfying

$$
\sum_{k \in \mathbb{Z}}|a(k)||k|^{\delta}<\infty \quad \text { for some } \delta>0 .
$$

Then the refinement equation (1.1) has at most one integrable solution up to a constant multiplication; see Daubechies and Lagarias [4].

When we consider solutions in $L_{2}(\mathbb{R})$, this uniqueness never holds. To see this, let $\phi \in L_{2}(\mathbb{R})$ satisfy the refinement equation (1.4) for some $2 \pi$-periodic function $\tilde{a}$. If $\tau(\xi)$ is an arbitrary measurable bounded function on $[-2 \pi, 2 \pi]$, then the function $\psi$ defined by its Fourier transform as

$$
\hat{\psi}(\xi)=\tau\left(2^{j} \xi\right) \hat{\phi}(\xi), \quad \xi \in\left[2^{-j} \pi, 2^{1-j} \pi\right) \cup\left(-2^{1-j} \pi,-2^{-j} \pi\right], \quad j \in \mathbb{Z},
$$

also satisfies the refinement equation (1.4) with the same mask.

However, if we require that $\hat{\phi}(\xi)$ is continuous at the origin, which is the case when $\phi \in L_{1}(\mathbb{R})$ and $\hat{\phi}(0) \neq 0$, then the solution is unique up to a constant multiplication.

Corollary 3. If $\phi \in L_{2}(\mathbb{R})$ satisfies (1.4) and its Fourier transform can be chosen to be continuous at the origin: $\lim _{\xi \rightarrow 0} \hat{\phi}(\xi)=\hat{\phi}(0) \neq 0$, then any solution $\psi \in L_{2}(\mathbb{R})$ of (1.4) with $\hat{\psi}$ continuous at the origin can be written as

$$
\psi(x)=\frac{\hat{\psi}(0)}{\hat{\phi}(0)} \phi(x) .
$$

Proof. By our assumption, for almost every $\xi$,

$$
\lim _{n \rightarrow \infty} \tilde{a}(\xi / 2) \cdots \tilde{a}\left(\xi / 2^{n}\right)=\frac{\hat{\phi}(\xi)}{\hat{\phi}(0)} .
$$

Therefore, for almost every $\xi$,

$$
\hat{\psi}(\xi)=\lim _{n \rightarrow \infty} \tilde{a}(\xi / 2) \cdots \tilde{a}\left(\xi / 2^{n}\right) \lim _{n \rightarrow \infty} \hat{\psi}\left(\xi / 2^{n}\right)=\frac{\hat{\psi}(0)}{\hat{\phi}(0)} \hat{\phi}(\xi) .
$$

The proof of Corollary 3 is complete.

As a consequence, if there is a solution $\phi \in L_{1}(\mathbb{R}) \cap L_{2}(\mathbb{R})$ to $(1.4)$ with $\hat{\phi}(0) \neq 0$, then all the other solutions in $L_{1}(\mathbb{R}) \cap L_{2}(\mathbb{R})$ are $c \phi(x)$. This extends the result of Daubechies and Lagarias [4].

\section{Inhomogeneous Refinement Equations}

In this section we study inhomogeneous refinement equations and characterize those solutions which are (homogeneously) refinable.

The inhomogeneous refinement equation was defined in [16] as

$$
\phi(x)=\sum_{k \in \mathbb{Z}} a(k) \phi(2 x-k)+F(2 x) .
$$


Here we are interested in $L_{2}$ solutions, so we assume that $F$ is a nonzero function in $L_{2}(\mathbb{R})$. Denote

$$
\tilde{a}(\xi)=\frac{1}{2} \sum_{k \in \mathbb{Z}} a(k) e^{-i k \xi}, \quad \xi \in \mathbb{R} .
$$

Then the inhomogeneous refinement equation (5.1) has an equivalent form in the Fourier domain:

$$
\hat{\phi}(2 \xi)=\tilde{a}(\xi) \hat{\phi}(\xi)+\hat{F}(\xi) / 2
$$

If $\phi \in L_{2}$ is a solution of (5.1) and $\phi$ is (homogeneously) refinable, then (1.4) holds for some $2 \pi$-periodic function $\tilde{\tau}$. Hence

$$
\hat{F}(\xi)=2(\tilde{\tau}(\xi)-\tilde{a}(\xi)) \hat{\phi}(\xi) .
$$

It follows from Theorem 1 that $F \in \bar{S}$.

Suppose now that $F$ and the mask $a$ are supported in $[0, N]$ for some $N \in \mathbb{N}$. Then $F \in S$ by Corollary 1. Also, $\phi \in S_{2}(F)$. Moreover, [16 tells us that $\phi$ is supported in $[0, N]$.

According to the analysis of Jia $[8$, for the function $F$ there exists a unique function $\psi \in L_{2}(\mathbb{R})$ (up to a constant multiplication), compactly supported in $[0, N]$ but not in $[1, N]$, such that its integer translates are linearly independent and, for some sequence $\{c(k)\}$,

$$
F(x)=\sum_{k=0}^{N-1} c(k) \psi(x-k) .
$$

By (1.4), $\phi \in S_{2}(F)=S_{2}(\psi)$. Corollary 1 implies again that $\psi$ is refinable.

By the linear independence of $\psi$ and the supports, there are sequences $\{b(k)\}$ and $\{d(k)\}$ such that

$$
\begin{gathered}
\phi(x)=\sum_{k=0}^{N-1} b(k) \psi(x-k), \\
\psi(x)=\sum_{k=0}^{N} d(k) \psi(2 x-k) .
\end{gathered}
$$

Taking Fourier transforms and using (5.1), (5.3), we have

$$
2 \tilde{b}(2 \xi) \tilde{d}(\xi) \hat{\psi}(\xi)=2 \tilde{a}(\xi) \tilde{b}(\xi) \hat{\psi}(\xi)+\tilde{c}(\xi) \hat{\psi}(\xi),
$$

which implies

$$
\tilde{b}(2 \xi) \tilde{d}(\xi)=\tilde{a}(\xi) \tilde{b}(\xi)+\tilde{c}(\xi) / 2, \quad \forall \xi \in \mathbb{R} .
$$

Moreover, the linear independence of $\psi$ provides (see Theorem 2.4 in [9])

$$
\hat{\psi}(0) \neq 0, \quad \tilde{d}(0)=1, \quad \text { and } \quad \tilde{d}(\pi)=0 .
$$

Conversely, we have

Theorem 6. Assume that the sequence $\{a(k)\}$ and the function $F \in L_{2}(\mathbb{R})$ are supported in $[0, N]$. Let $\psi \in L_{2}(\mathbb{R})$ be a function, compactly supported in $[0, N]$ but not in $[1, N]$, such that its integer translates are linearly independent, and (5.3) holds. Then (5.1) has a refinable solution $\phi \in L_{2}(\mathbb{R})$ if and only if $\psi$ is refinable, $\hat{\psi}(0) \neq 0$ and the equation (5.4) is solvable for some sequence $\{b(k)\}$ supported in 
$[0, N-1]$, where $\tilde{d}(\xi)$ is the symbol of the refinement mask of the function $\psi$ with $\tilde{d}(0)=1$ and $\tilde{d}(\pi)=0$.

Observe that (5.4) is a system of linear equations whose solvability can be easily checked.

\section{Applications to Multiple Refinable Functions}

In this section we apply Theorem 6 to a study of some examples of multiple refinable functions. For the general theory and more examples of multiple refinable functions, we refer the reader to [5], [6], [10], [11], [12], [15], [18].

The first example was introduced by Geronimo, Hardin and Massopust [5]. Consider the matrix refinement equation

$$
\Phi(x)=\sum_{k \in \mathbb{Z}} a_{k} \Phi(2 x-k) .
$$

Here $\Phi(x)=\left(\phi_{1}(x), \phi_{2}(x)\right)^{T}$ and $\left\{a_{k}\right\}$ is supported in $[0,3]$ with

$$
\begin{array}{lll}
a_{0}=\left[\begin{array}{cc}
h_{1} & 1 \\
h_{2} & h_{3}
\end{array}\right], & a_{1}=\left[\begin{array}{ll}
h_{1} & 0 \\
h_{4} & 1
\end{array}\right], \\
a_{2}=\left[\begin{array}{cc}
0 & 0 \\
h_{4} & h_{3}
\end{array}\right], & a_{3}=\left[\begin{array}{cc}
0 & 0 \\
h_{2} & 0
\end{array}\right] .
\end{array}
$$

The matrix entries involve a parameter $s$ :

$$
\begin{array}{cc}
h_{1}=-\frac{s^{2}-4 s-3}{2(s+2)}, & h_{2}=-\frac{3\left(s^{2}-1\right)\left(s^{2}-3 s-1\right)}{4(s+2)^{2}}, \\
h_{3}=\frac{3 s^{2}+s-1}{2(s+2)}, & h_{4}=-\frac{3\left(s^{2}-1\right)\left(s^{2}-s+3\right)}{4(s+2)^{2}} .
\end{array}
$$

When $|s|<1$, the matrix refinement equation (6.1) has a continuous solution $\Phi$ with $\hat{\phi}_{1}(0)=1$ and $\hat{\phi}_{2}(0)=(s-1)^{2} /(s+2)$. Moreover, supp $\phi_{1}=[0,1]$ and $\operatorname{supp} \phi_{2}=[0,2]$.

Applying Theorem 6, we conclude that neither $\phi_{1}$ nor $\phi_{2}$ is refinable.

Example 2. Let $|s|<1$, and let $\Phi(x)=\left(\phi_{1}(x), \phi_{2}(x)\right)^{T}$ be the continuous solution of $(6.1)$ with $\hat{\phi}_{1}(0)=1$ and $\hat{\phi}_{2}(0)=(s-1)^{2} /(s+2)$. Then neither $\phi_{1}$ nor $\phi_{2}$ is refinable.

Proof. The first component of (6.1) is an inhomogeneous refinement equation:

$$
\phi_{1}(x)=h_{1} \phi_{1}(2 x)+h_{1} \phi_{1}(2 x-1)+\phi_{2}(2 x) .
$$

It is proved in [12] that the integer translates of $\phi_{2}$ are linearly independent. Hence we can take $N=2, F=\psi=\phi_{2}$, and $\tilde{c} \equiv 1$. Under the restrictions $\tilde{d}(0)=1$ and $\tilde{d}(\pi)=0$, we find that the equation (5.4) is not solvable. Therefore, $\phi_{1}$ is not refinable.

The second component of (6.1) is another inhomogeneous refinement equation,

$$
\phi_{2}(x)=h_{3} \phi_{2}(2 x)+\phi_{2}(2 x-1)+h_{3} \phi_{2}(2 x-2)+F(2 x),
$$

where

$$
F(x)=h_{2} \phi_{1}(x)+h_{4} \phi_{1}(x-1)+h_{4} \phi_{1}(x-2)+h_{2} \phi_{1}(x-3) .
$$


Take $N=4$ and $\psi=\phi_{1}$, since the integer translates of $\phi_{1}$ are linearly independent [12]. If $\phi_{2}$ is refinable, then Theorem 6 shows that $\phi_{1}=\psi$ is also refinable, which is a contradiction. Thus, $\phi_{2}$ is not refinable, either.

Our second example is taken from [10, [11, 12]. Let $\left\{a_{k}\right\}$ be supported in $[0,2]$ with

$$
a_{0}=\left[\begin{array}{cc}
\frac{1}{2} & \frac{s}{2} \\
t & \lambda
\end{array}\right], \quad a_{1}=\left[\begin{array}{cc}
1 & 0 \\
0 & \mu
\end{array}\right], \quad \text { and } \quad a_{2}=\left[\begin{array}{cc}
\frac{1}{2} & -\frac{s}{2} \\
-t & \lambda
\end{array}\right] .
$$

Here $s, t, \lambda, \mu$ are real parameters. We assume that $|2 \lambda+\mu|<2$. Then there exists a unique distributional solution $\Phi(x)=\left(\phi_{1}(x), \phi_{2}(x)\right)^{T}$ of $(6.1)$ with $\hat{\Phi}(0)=(1,0)^{T}$ supported in $[0,2]$. The distribution $\phi_{1}(x)$ is symmetric about 1 , and $\phi_{2}(x)$ is antisymmetric about 1. It was proved in [10, Example 4.3] that the shifts of $\phi_{1}$ and $\phi_{2}$ reproduce all quadratic polynomials if and only if

$$
t \neq 0, \quad \mu=1 / 2, \quad \text { and } \quad \lambda=1 / 4+2 s t .
$$

In this case, the condition $|2 \lambda+\mu|<2$ reduces to $-3 / 4<s t<1 / 4$, and it is verified in [11], [12] that the solution is continuous.

Example 3. Let $\left\{a_{k}\right\}$ be the mask given in (6.3) and (6.4) with $-3 / 4<s t<1 / 4$. Let $\Phi(x)=\left(\phi_{1}(x), \phi_{2}(x)\right)^{T}$ be the continuous solution of $(6.1)$ with $\hat{\Phi}(0)=(1,0)^{T}$. Then $\phi_{1}$ is refinable if and only if $s=0$, while $\phi_{2}$ is never refinable.

Proof. First, we consider the case $s \neq 0$. In this case, it is proved in $[12$ that the integer translates of $\phi_{1}$ and $\phi_{2}$ are linearly independent.

For $\phi_{1}$, the first component of (6.1) is an inhomogeneous refinement equation,

$$
\phi_{1}(x)=\phi_{1}(2 x) / 2+\phi_{1}(2 x-1)+\phi_{1}(2 x-2) / 2+F(x),
$$

where $F(x)=s \phi_{2}(x) / 2-s \phi_{2}(x-2) / 2$. Let $N=4$ and $\psi=\phi_{2}$. If $\phi_{1}$ is refinable, then Theorem 6 shows that $\hat{\phi}_{2}(0)=\hat{\psi}(0) \neq 0$, which is a contradiction.

The function $\phi_{2}$ is trivially not refinable, since otherwise $\hat{\phi}_{2}(0) \neq 0$ by 9 , Theorem 2.4].

Second, we investigate the case $s=0$. Then $\phi_{1}$ is refinable, since the first component of (6.1) reduces to a homogeneous equation for $\phi_{1}$. In fact, $\phi_{1}$ is the hat function on $[0,2]$.

To consider $\phi_{2}$, the second component of $(6.1)$ is

$$
\phi_{2}(x)=\phi_{2}(2 x) / 4+\phi_{2}(2 x-1) / 2+\phi_{2}(2 x-2) / 4+F(2 x),
$$

where $F(x)=t \phi_{1}(x)-t \phi_{1}(x-2)$. By Theorem 1 in [16], the solution to this equation is unique. Let $N=4$ and $\psi=\phi_{1}$. Then $\tilde{d}(\xi)=\left(1+e^{-i \xi}\right)^{2} / 4$ and $\tilde{c}(\xi)=t\left(1-e^{-i 2 \xi}\right) / 2$, and the solvability of equation (5.4) would imply $t=0$, which is a contradiction. Therefore, $\phi_{2}$ is never refinable.

The explicit formula for the solution $\Phi(x)$ in the special case $s=3 / 2, t=-1 / 8$, $\lambda=-1 / 8$, and $\mu=1 / 2$ was given by Heil, Strang, and Strela [6]. In this case, $\Phi(x)$ is supported on $[0,2]$ :

$$
\phi_{1}(x)= \begin{cases}x^{2}(-2 x+3) & \text { for } 0 \leq x \leq 1, \\ (2-x)^{2}(2 x-1) & \text { for } 1<x \leq 2\end{cases}
$$


and

$$
\phi_{2}(x)= \begin{cases}x^{2}(x-1) & \text { for } 0 \leq x \leq 1 \\ (2-x)^{2}(x-1) & \text { for } 1<x \leq 2\end{cases}
$$

\section{Fully Refinable Functions}

A function $\phi \in L_{2}(\mathbb{R})$ is fully refinable if for every $t \in \mathbb{R}$, the shifted function $\phi_{t}(x):=\phi(x-t)$ is refinable. It is shown in [17] that Meyer's well-known scaling function [13] is fully refinable.

Let $\phi$ be a refinable function in $L_{2}(\mathbb{R})$ and $t \in \mathbb{R}$. Then $K_{j}\left(\phi_{t}\right)=K_{j}(\phi)$ for $j \in \mathbb{N} \cup\{0\}$. Theorem 3 tells us that $\phi_{t}$ is refinable if and only if $\phi_{t} \in \bar{S}$. By Theorem 1, this is equivalent to

$$
\hat{\phi}\left(2^{j}(\xi+2 k \pi)\right) \hat{\phi}(\xi)\left(e^{-i t\left(2^{j}-1\right) 2 k \pi}-1\right)=0
$$

for any $j, k \in \mathbb{N}$, almost everywhere in $\xi$.

Thus, a translate $\phi_{t}$ of a compactly supported refinable function $\phi \in L_{2}(\mathbb{R})$ is not refinable unless $t$ is an integer.

Moreover, if $\phi$ is fully refinable, then up to a null set $\hat{\phi}(\xi) \neq 0$ implies $\hat{\phi}(\xi / 2) \neq 0$, and hence

$$
\hat{\phi}(\xi+4 k \pi)=\hat{\phi}(2(\xi / 2+2 k \pi))=0, \quad \forall k \in \mathbb{Z} \backslash\{0\} .
$$

This shows that the measure of the support of $\hat{\phi}$ is not greater than $4 \pi$.

However, Theorem 4 tells that every refinable function in $X_{\frac{4}{3} \pi}$ is fully refinable.

\section{REFERENCES}

[1] C. de Boor, R. DeVore and A. Ron, Approximation from shift-invariant subspaces of $L_{2}(\mathbb{R})$, Trans. Amer. Math. Soc. 341 (1994), 787-806. MR 94d:41028

[2] J. O. Chapa, Matched wavelet construction and its application to target detection, Ph.D. thesis, Rochester Institute of Technology, 1995.

[3] I. Daubechies, Ten Lectures on Wavelets, SIAM, 1992. MR 93e:42045

[4] I. Daubechies and J. C. Lagarias, Two-scale difference equations: I. Existence and global regularity of solutions, SIAM J. Math. Anal. 22 (1991), 1388-1410. MR 92d:39001

[5] J. S. Geronimo, D. P. Hardin, and P. R. Massopust, Fractal functions and wavelet expansions based on several functions, J. Approx. Theory 78 (1994), 373-401. MR 95h:42033

[6] C. Heil, G. Strang, and V. Strela, Approximation by translates of refinable functions, Numer. Math. 73 (1996), 75-94. MR 97c:65033

[7] H. Helson, Lectures on Invariant Subspaces, Academic Press, New York, 1964. MR 30:1409

[8] R. Q. Jia, Shift-invariant spaces on the real line, Proc. Amer. Math. Soc. 125 (1997), 785-793. MR 97e:41039

[9] R. Q. Jia and C. A. Micchelli, Using the refinement equations for the construction of prewavelets II: Power of two, Curves and Surfaces" (P. J. Laurent, A. Le Méhauté, and L. L. Schumaker, Eds.), Academic Press, New York, 1991, pp. 209-246. MR 93e:65024

[10] R. Q. Jia, S. D. Riemenschneider, and D. X. Zhou, Approximation by multiple refinable functions, Canadian J. Math. 49 (1997), 944-962. MR 99f:39036

[11] R. Q. Jia, S. D. Riemenschneider, and D. X. Zhou, Vector subdivision schemes and multiple wavelets, Math. Comp. 67 (1998), 1533-1563. MR 99d:42062

[12] R. Q. Jia, S. D. Riemenschneider, and D. X. Zhou, Smoothness of multiple refinable functions and multiple wavelets, SIAM J. Matrix Anal. Appl. 21 (1999), 1-28. CMP 2000:01

[13] Y. Meyer, Wavelets and Operators, Cambridge University Press, 1993. MR 94f:42001

[14] G. Strang and T. Nguyen, Wavelets and Filter Banks, Wellesley-Cambridge Press, 1996. MR 98b:94003

[15] G. Strang and V. Strela, Orthogonal multiwavelets with vanishing moments, Optical Eng. 33 (1994), 2104-2107. 
[16] G. Strang and D. X. Zhou, Inhomogeneous refinement equations, J. Fourier Anal. Appl. 4 (1998), 733-747. MR 99m:42056

[17] D. X. Zhou, Construction of real-valued wavelets by symmetry, J. Approx. Theory 81 (1995), 323-331. MR 96m:42047

[18] D. X. Zhou, Existence of multiple refinable distributions, Michigan Math. J. 44 (1997), 317329. MR 99a:41021

Department of Mathematics, Massachusetts Institute of Technology, Cambridge, MASSACHUSETTS 02139

E-mail address: gs@math.mit.edu

Department of Mathematics, City University of Hong Kong, Tat Chee Avenue, KowLOON, Hong Kong, P. R. China

E-mail address: mazhou@math.cityu.edu.hk 